\title{
Certain Results on Ricci Solitons in Trans-Sasakian Manifolds
}

\author{
C. S. Bagewadi and Gurupadavva Ingalahalli \\ Department of Mathematics, Kuvempu University, Shankaraghatta, Shimoga, Karnataka 577 451, India
}

Correspondence should be addressed to C. S. Bagewadi; prof_bagewadi@yahoo.co.in

Received 29 September 2012; Revised 3 January 2013; Accepted 5 January 2013

Academic Editor: Andrea Pascucci

Copyright (C) 2013 C. S. Bagewadi and G. Ingalahalli. This is an open access article distributed under the Creative Commons Attribution License, which permits unrestricted use, distribution, and reproduction in any medium, provided the original work is properly cited.

We study and obtain results on Ricci solitons in trans-Sasakian manifolds satisfying $R(\xi, X) \cdot \widetilde{C}=0, P(\xi, X) \cdot \widetilde{C}=0, H(\xi, X) \cdot S=0$, and $\widetilde{C}(\xi, X) \cdot S=0$, where $\widetilde{C}, P$, and $H$ are quasiconformal, projective, and conharmonic curvature tensors.

\section{Introduction}

During 1982, Hamilton [1] made the fundamental observation that Ricci flow is an excellent tool for simplifying the structure of a manifold. It is a process which deforms the metric of a Riemannian manifold analogous to the diffusion of heat there by smoothing out the regularity in the metric. It is given by $(\partial g / \partial t)=-2$ Ric $g$.

1.1. Example. (i) If the manifold is an Euclidean space or more generally Ricci-flat, then Ricci flow leaves the metric unchanged. Conversely, any metric unchanged by Ricci flow is Ricci-flat.

(ii) Let $M^{n}=S^{n}$ and $g_{S^{n}}$ the standard metric on the unit $n$-sphere in the Euclidean space. If $g_{0}=r_{0}^{2} g_{S^{n}}$ for some $r_{0}>0$ ( $r_{0}$ is the radius), then $g(t)=\left(r_{0}^{2}-2(n-1) t\right) g_{S^{n}}$ is a solution. So the Ricci flow with $g(0)=g_{0}$ collapses to a point at time $t=r_{0} / 2(n-1)$.

Ricci solitons move under the Ricci flow simply by diffeomorphisms of the initial metric, that is, they are stationary points of the Ricci flow in space of metrics on $M$. Here the metric $g(t)$ is the pull back of the initial metric $g(0)$ by a 1parameter family of diffeomorphisms $\phi_{t}$ generated by a vector field on a manifold $M$.

Let $\sigma(t)$ be a smooth function of time. Since $\phi_{t}: M \rightarrow M$ is a diffeomorphism and $g(t)$ is a Riemannian metric on $M$ (codomain), then by definition of pull back $\phi_{t}^{*} g(t)$ is a metric on $M$ (domain).
Set $\widetilde{g}(t)=\sigma(t) \phi_{t}^{*}(g(t))$; then we have [2]

$$
\frac{\partial \widetilde{g}}{\partial t}=\sigma^{\prime}(t) \phi_{t}^{*}(g(t))+\sigma(t) \phi_{t}^{*} \frac{\partial g}{\partial t}+\sigma(t) \phi_{t}^{*}\left(L_{X} g\right)
$$

(This follows from the definition of the Lie derivative.)

Suppose we have a metric $g_{0}$, a vector field $Y$, and $\lambda \in R$ (all independent of time) such that

$$
L_{Y} g_{0}+2 \operatorname{Ric} g_{0}+2 \lambda g_{0}=0 .
$$

If we choose $g(t)=g_{0}, \sigma(t)=1-2 \lambda t$, and $X(t)=(1 / \sigma(t)) Y$, then it gives a family of diffeomorphisms $\phi_{t}$ with $\phi_{0}$ identity; then using (2) in (1), $\widetilde{g}$ defined above is a Ricci flow with $g(0)=g_{0}$, that is,

$$
\frac{\partial \widetilde{g}}{\partial t}=-2 \operatorname{Ric} \tilde{g} .
$$

Hence, $L_{X} g_{0}+2 \mathrm{Ric} g_{0}+2 \lambda g_{0}=0$ is a solution of the Ricci flow and is known as a Ricci soliton.

Definition 1 (see [3]). A Ricci soliton on a Riemannian manifold is defined by

$$
L_{X} g+2 S+2 \lambda g=0 .
$$

It is said to be shrinking, steady, or expanding according to $\lambda<0, \lambda=0$, and $\lambda>0$. 
1.2. Example. (1) Hamilton Cigar Soliton [4]. Let $M=R^{2}$, and $\phi_{t}: R^{2} \rightarrow R^{2}$ defined by $\phi_{t}(x, y)=\left(e^{-2 t} x, e^{-2 t} y\right)$ forms a family of a one-parameter group of diffeomorphisms. The vector field $X$ generated by $\left\{\phi_{t}\right\}$ is $X=-2(x(\partial / \partial x)+y(\partial / \partial y))$. The metric $g_{0}$ is obtained as $g_{0}=\left(d x^{2}+d y^{2}\right) /\left(1+x^{2}+\right.$ $\left.y^{2}\right), \tilde{g}(t)=\phi_{t}^{*}\left(g_{0}\right)=\left(d x^{2}+d y^{2}\right) /\left(e^{4 t}+x^{2}+y^{2}\right)$, Ric $g_{0}=$ $\left(2 /\left(1+x^{2}+y^{2}\right)\right) g_{0}, L_{X} g_{0}=\left(4 /\left(1+x^{2}+y^{2}\right)\right) g_{0}$. Using (4), we have $\lambda=0$. Hence, this Ricci soliton is steady and is called cigar soliton as it is asymptotic to a flat cylinder at infinity.

(2) Cylinder Shrinking Soliton [4]. Consider the product of the sphere with a like $\left\{\left(S^{2} \times R, g(t)\right): t \in(-\infty, 0)\right\}$, where $g(t)=2|t| g_{S^{2}}+d r^{2}$. Its Ricci tensor is given by $\operatorname{Ric}(g(t))=$ $g_{S^{2}}=(1 / 2|t|) g(t)-(1 / 2|t|) d r^{2}$. If we set $\phi(\theta, r, t)=\left(r^{2} / 4|t|\right)$, $\theta \in S^{2}, r \in R, t<0$, then $2 \operatorname{Ric} g(t)+2 t \nabla \phi g(t)+(1 / t) g(t)=0$, $\lambda=1 / t<0$. Hence $g(t)$ is a shrinking soliton.

In [3], Sharma obtained some interesting results on Ricci solitons in K-contact manifolds. Cǎlin and Crasmareanu [5] extended the Eisenhart problem to Ricci solitons in $f$ Kenmotsu manifolds. They studied the case of $f$-Kenmotsu manifolds satisfying a special condition called regular and showed that a symmetric parallel tensor field of second order is a constant multiple of the Riemannian metric. Using this result, they obtained the results on Ricci solitons concerned in $f$-Kenmotsu manifolds and 3 -dimensional $\beta$-Kenmotsu manifolds.

In $[6,7]$, Bagewadi and Ingalahalli studied Ricci solitons in $\alpha$-Sasakian and Lorentzian $\alpha$-Sasakian manifolds and proved that a symmetric parallel tensor field of second order is a constant multiple of the Riemannian metric; based on these they obtained the results on Ricci Solitons concerned in these manifolds. In [8], Tripathi obtained some results on Ricci solitons in contact metric manifolds. In [9], Nagaraja and Premalatha used semisymmetric conditions in Kenmotsu manifolds and obtained reuslts on Ricci solitons in Kenmotsu manifolds.

Motivated by all this work in this paper we are studying the Ricci solitons in trans-Sasakian manifolds.

Trans-Sasakian manifolds arose in a natural way from the classification of almost contact metric structures, and they appear as a natural generalization of both Sasakian and Kenmotsu manifolds. In [10], Gray-Hervella classification of almost Hermite manifolds appears as a class $W_{4}$ of Hermitian manifolds which are closely related to locally conformally Kähler manifolds. An almost contact metric structure on a manifold $M$ is called a trans-Sasakian structure [11] if the product manifold $M \times R$ belongs to the class $W_{4}$. The class $C_{6} \oplus C_{5}$ [12] coincides with the class of trans-Sasakian structures of type $(\alpha, \beta)$. The local nature of the two subclasses $C_{5}$ and $C_{6}$ of trans-Sasakian structures is characterized completely. It is known that [13] trans-Sasakian structures of type $(0,0),(\alpha, 0)$, and $(0, \beta)$, are cosymplectic, $\alpha$-Sasakian, and $\beta$-Kenmotsu, respectively, where $\alpha, \beta \in R$. In [12], Marrero has shown that a trans-Sasakian manifold of dimension $n \geq 5$ is either cosymplectic or $\alpha$-Sasakian or $\beta$ Kenmotsu manifold. Later many authors worked in this topic, trans-Sasakian manifolds, like Blair and Oubiña [14], Janssens and Vanhecke [13], Bagewadi and Venkatesha [15], U. De and
K. De [16], De and Tripathi [17], Shaikh et al. [18], Nagaraja et al. [19]. Now we move on to the preliminaries.

\section{Preliminaries}

An $n$-dimensional differential manifold $M$ is said to be an almost contact metric manifold $[20]$ if it admits a $(1,1)$ tensor field $\phi$, a vector field $\xi$, a 1 -form $\eta$, and a Riemannian metric $g$, satisfying

$$
\begin{gathered}
\phi^{2}=-I+\eta \otimes \xi, \quad \eta(\xi)=1, \\
\eta \circ \phi=0, \quad \phi \xi=0, \\
g(\phi X, \phi Y)=g(X, Y)-\eta(X) \eta(Y), \quad g(X, \xi)=\eta(X),
\end{gathered}
$$

for all vector fields $X, Y$ on $M$.

An almost contact metric manifold $M(\phi, \xi, \eta, g)$ is said to be trans-Sasakian manifold if $(M \times R, J, G)$ belongs to the class $W_{4}$ of the Hermitian manifolds [12], where $J$ is the almost complex structure of $M \times R$ defined by

$$
J\left(Z, \frac{f d}{d t}\right)=\left(\phi Z-f \xi, \frac{\eta(Z) d}{d t}\right),
$$

for all vector fields $Z$ on $M$ and smooth function $f$ on $M \times R$, and $G$ is the product metric on $M \times R$. This may be expressed by the following condition [13]:

$$
\begin{aligned}
\left(\nabla_{X} \phi\right) Y= & \alpha(g(X, Y) \xi-\eta(Y) X) \\
& +\beta(g(\phi X, Y) \xi-\eta(Y) \phi X),
\end{aligned}
$$

where $\alpha$ and $\beta$ are scalar functions on $M$, and such a structure is said to be the trans-Sasakian structure of type $(\alpha, \beta)$. From (7), we have

$$
\begin{gathered}
\nabla_{X} \xi=-\alpha \phi X+\beta(X-\eta(X) \xi) \\
\left(\nabla_{X} \eta\right)(Y)=-\alpha g(\phi X, Y)+\beta g(\phi X, \phi Y) .
\end{gathered}
$$

Note 1. (1) For some smooth functions $\alpha$ and $\beta$, if $\alpha \neq 0$ and $\beta=0, \alpha=0$, and $\beta \neq 0$, then it reduces to $\alpha$-Sasakian and $\beta$-Kenmotsu manifold, respectively.

(2) If $\alpha$ and $\beta$ are scalars and $\alpha=1$ and $\beta=0, \alpha=0$ and $\beta=1$, then it reduces to Sasakian and Kenmotsu manifold, respectively.

(3) If $\alpha=0$ and $\beta=0$, then it reduces to cosymplectic manifold, respectively. 
In a trans-Sasakian manifold, we have [17]

$$
\begin{aligned}
R(X, Y) \xi= & \left(\alpha^{2}-\beta^{2}\right)[\eta(Y) X-\eta(X) Y] \\
& -(X \alpha) \phi Y-(X \beta) \phi^{2} Y \\
& +2 \alpha \beta[\eta(Y) \phi X-\eta(X) \phi Y] \\
& +(Y \alpha) \phi X+(Y \beta) \phi^{2} X \\
R(\xi, X) \xi= & \left(\alpha^{2}-\beta^{2}-\xi \beta\right)(\eta(X) \xi-X) \\
\eta(R(X, Y) Z)=\left(\alpha^{2}-\beta^{2}\right) & \cdot[g(Y, Z) \eta(X)-g(X, Z) \eta(Y)]+2 \alpha \beta \\
\cdot & {[g(\phi Y, Z) \eta(X)-g(\phi X, Z) \eta(Y)] } \\
+ & {[(X \alpha) g(\phi Y, Z)-(Y \alpha) g(\phi X, Z)] } \\
+ & {\left[(X \beta) g\left(\phi^{2} Y, Z\right)-(Y \beta) g\left(\phi^{2} X, Z\right)\right] } \\
& \\
& 2 \alpha \beta+\xi \alpha=0,
\end{aligned}
$$

where $\alpha, \beta$ are functions and $R$ is the Riemannian curvature tensor. From (8) we have

$$
\left(\mathscr{L}_{\xi} g\right)(X, Y)=2 \beta[g(X, Y)-\eta(X) \eta(Y)] .
$$

From (4) and (14), we get

$$
S(X, Y)=-(\lambda+\beta) g(X, Y)+\beta \eta(X) \eta(Y) .
$$

The above equation yields

$$
\begin{gathered}
Q X=-(\lambda+\beta) X+\beta \eta(X) \xi, \\
S(X, \xi)=-\lambda \eta(X), \\
r=-\lambda n-(n-1) \beta,
\end{gathered}
$$

where $Q$ is the Ricci operator and $r$ is the scalar curvature on M.

Remark 2. Since our study deals with Ricci solitons of transSasakian manifolds and by Definition 1 , it is known that $\lambda$ is a scalar quantity. Further, from (18) it is seen that $\lambda$ is related to $\beta$. Hence in the calculations of our results which will be proved in the following it is necessary to assume $\alpha$ and $\beta$ as simply scalar quantities.

2.1. Example for 3-Dimensional Trans-Sasakian Manifold. We consider the 3-dimensional manifold $M=\left\{(x, y, z) \in R^{3}\right.$; $z \neq 0\}$, where $(x, y, z)$ are the standard co-ordinates in $R^{3}$. Let $\left\{E_{1}, E_{2}, E_{3}\right\}$ be linearly independent global frame field on $M$ given by

$$
E_{1}=z\left(\frac{\partial}{\partial x}+y \frac{\partial}{\partial z}\right), \quad E_{2}=z \frac{\partial}{\partial y}, \quad E_{3}=\frac{\partial}{\partial z}
$$

Let $g$ be the Riemannian metric defined by $g\left(E_{1}, E_{2}\right)=$ $g\left(E_{2}, E_{3}\right)=g\left(E_{1}, E_{3}\right)=0, g\left(E_{1}, E_{1}\right)=g\left(E_{2}, E_{2}\right)=g\left(E_{3}\right.$, $\left.E_{3}\right)=1$, where $g$ is given by

$$
g=\frac{1}{z^{2}}\left[\left(1-y^{2} z^{2}\right) d x \otimes d x+d y \otimes d y+z^{2} d z \otimes d z\right]
$$

The $(\phi, \xi, \eta)$ is given by $\eta=d z-y d x, \xi=E_{3}=\partial / \partial z, \phi E_{1}=$ $E_{2}, \phi E_{2}=-E_{1}, \phi E_{3}=0$. The linearity property of $\phi$ and $g$ yields that $\eta\left(E_{3}\right)=1, \phi^{2} U=-U+\eta(U) E_{3}, g(\phi U, \phi W)=$ $g(U, W)-\eta(U) \eta(W)$, for any vector fields $U, W$ on $M$. By the definition of Lie bracket, we have

$$
\begin{gathered}
{\left[E_{1}, E_{2}\right]=y E_{2}-z^{2} E_{3},} \\
{\left[E_{1}, E_{3}\right]=-\frac{1}{z} E_{1}, \quad\left[E_{2}, E_{3}\right]=-\frac{1}{z} E_{2} .}
\end{gathered}
$$

Let $\nabla$ be the Levi-Civita connection with respect to the above metric $g$ given by the Koszula formula

$$
\begin{aligned}
2 g\left(\nabla_{X} Y, Z\right)= & X(g(Y, Z))+Y(g(Z, X)) \\
& -Z(g(X, Y))-g(X,[Y, Z]) \\
& -g(Y,[X, Z])+g(Z,[X, Y]) .
\end{aligned}
$$

Then,

$$
\begin{gathered}
\nabla_{E_{1}} E_{3}=-\frac{1}{z} E_{1}+\frac{1}{2} z^{2} E_{2}, \\
\nabla_{E_{2}} E_{3}=-\frac{1}{z} E_{2}-\frac{1}{2} z^{2} E_{1}, \quad \nabla_{E_{3}} E_{3}=0, \\
\nabla_{E_{2}} E_{2}=y E_{1}+\frac{1}{z} E_{3}, \quad \nabla_{E_{1}} E_{2}=-\frac{1}{2} z^{2} E_{3}, \\
\nabla_{E_{2}} E_{1}=\frac{1}{2} z^{2} E_{3}-y E_{2}, \quad \nabla_{E_{1}} E_{1}=\frac{1}{z} E_{3}, \\
\nabla_{E_{3}} E_{2}=-\frac{1}{2} z^{2} E_{1}, \quad \nabla_{E_{3}} E_{1}=\frac{1}{2} z^{2} E_{2} .
\end{gathered}
$$

The tangent vectors $X$ and $Y$ to $M$ are expressed as linear combinations of $E_{1}, E_{2}, E_{3}$, that is, $X=\Sigma_{i=1}^{3} a_{i} E_{i}$ and $Y=$ $\sum_{i=1}^{3} b_{i} E_{i}$, where $a_{i}, b_{i}(i=1,2,3)$ are scalars. Clearly $(\phi, \xi, \eta, g)$ and $X, Y$ satisfy (5), (7), and (8) with $\alpha=-(1 / 2) z^{2} \neq 0$ and $\beta=-1 / z \neq 0$. Thus $M$ is a trans-Sasakian manifold.

2.2. Example for 3-Dimensional $\alpha$-Sasakian Manifolds. Let $M=\left\{(x, y, z) \in R^{3}\right\}$. Let $\left(E_{1}, E_{2}, E_{3}\right)$ be linearly independent vector fields given by

$$
E_{1}=k \frac{\partial}{\partial y}, \quad E_{2}=k\left(\frac{\partial}{\partial x}+2 y \frac{\partial}{\partial z}\right), \quad E_{3}=\frac{\partial}{\partial z} .
$$

Let $g$ be the Riemannian metric defined by $g\left(E_{1}, E_{2}\right)=$ $g\left(E_{2}, E_{3}\right)=g\left(E_{1}, E_{3}\right)=0, g\left(E_{1}, E_{1}\right)=g\left(E_{2}, E_{2}\right)=g\left(E_{3}\right.$, $\left.E_{3}\right)=1$, where $g$ is given by

$$
g=\frac{1}{k^{2}}\left[\left(1-4 k^{2} y^{2}\right) d x \otimes d x+d y \otimes d y+k^{2} d z \otimes d z\right] .
$$


The $(\phi, \xi, \eta)$ is given by

$$
\begin{gathered}
\eta=d z-2 y d x, \quad \xi=E_{3}=\frac{\partial}{\partial z}, \\
\phi E_{1}=E_{2}, \quad \phi E_{2}=-E_{1}, \quad \phi E_{3}=0 .
\end{gathered}
$$

The linearity property of $\phi$ and $g$ yields that $\eta\left(E_{3}\right)=1, \phi^{2} U=$ $-U+\eta(U) E_{3}, g(\phi U, \phi W)=g(U, W)-\eta(U) \eta(W)$, for any vector fields $U, W$. By the definition of Lie bracket, we have

$$
\left[E_{1}, E_{2}\right]=2 k^{2} E_{3}, \quad\left[E_{1}, E_{3}\right]=\left[E_{2}, E_{3}\right]=0 .
$$

Let $\nabla$ be the Levi-Civita connection; with respect to the above metric $g$ given by Koszula formula (22) and by virtue of it we have

$$
\begin{gathered}
\nabla_{E_{1}} E_{1}=0, \quad \nabla_{E_{2}} E_{2}=0, \quad \nabla_{E_{3}} E_{3}=0, \\
\nabla_{E_{1}} E_{2}=k^{2} E_{3}, \quad \nabla_{E_{2}} E_{1}=-k^{2} E_{3}, \\
\nabla_{E_{2}} E_{3}=k^{2} E_{1}, \quad \nabla_{E_{1}} E_{3}=-k^{2} E_{2}, \\
\nabla_{E_{3}} E_{1}=-k^{2} E_{2}, \quad \nabla_{E_{3}} E_{2}=k^{2} E_{1} .
\end{gathered}
$$

The tangent vectors $X$ and $Y$ to $M$ are expressed as linear combinations of $E_{1}, E_{2}, E_{3}$, that is, $X=\sum_{i=1}^{3} a_{i} E_{i}$ and $Y=$ $\sum_{i=1}^{3} b_{i} E_{i}$, where $a_{i}$ and $b_{i}(i=1,2,3)$ are scalars. This becomes $\alpha$-Sasakian manifold with $\alpha=k^{2}$.

2.3. Example for 3-Dimensional $\beta$-Kenmotsu Manifold. Let $M=\left\{(x, y, z) \in R^{3}\right\}$. Let $\left(E_{1}, E_{2}, E_{3}\right)$ be linearly independent vector fields given by

$$
\begin{gathered}
E_{1}=e^{-k z}\left(\frac{\partial}{\partial x}+\frac{\partial}{\partial y}\right), \quad E_{2}=e^{-k z}\left(-\frac{\partial}{\partial x}+\frac{\partial}{\partial y}\right), \\
E_{3}=\frac{\partial}{\partial z} .
\end{gathered}
$$

Let $g$ be the Riemannian metric defined by $g\left(E_{1}, E_{2}\right)=g\left(E_{2}\right.$, $\left.E_{3}\right)=g\left(E_{1}, E_{3}\right)=0, g\left(E_{1}, E_{1}\right)=g\left(E_{2}, E_{2}\right)=g\left(E_{3}, E_{3}\right)=1$, where $g$ is given by

$$
g=\frac{e^{2 k z}}{2}(d x \otimes d x+d y \otimes d y)+d z \otimes d z
$$

The $(\phi, \xi, \eta)$ is given by

$$
\begin{gathered}
\eta=d z, \quad \xi=E_{3}=\frac{\partial}{\partial z} \\
\phi E_{1}=E_{2}, \quad \phi E_{2}=-E_{1}, \quad \phi E_{3}=0 .
\end{gathered}
$$

The linearity property of $\phi$ and $g$ yields that $\eta\left(E_{3}\right)=1, \phi^{2} U=$ $-U+\eta(U) E_{3}, g(\phi U, \phi W)=g(U, W)-\eta(U) \eta(W)$, for any vector fields $U, W$ on $M$. By the definition of Lie bracket, we have

$$
\left[E_{1}, E_{2}\right]=0, \quad\left[E_{2}, E_{3}\right]=k E_{2}, \quad\left[E_{1}, E_{3}\right]=k E_{1} .
$$

Let $\nabla$ be the Levi-Civita connection; with respect to above metric $g$ given by Koszula formula (22) and by virtue of it, we have

$$
\begin{array}{lcc}
\nabla_{E_{1}} E_{3}=k E_{1}, & \nabla_{E_{2}} E_{3}=k E_{2}, & \nabla_{E_{3}} E_{3}=0, \\
\nabla_{E_{1}} E_{2}=0, & \nabla_{E_{2}} E_{2}=-k E_{3}, & \nabla_{E_{3}} E_{2}=0, \\
\nabla_{E_{1}} E_{1}=-k E_{3}, & \nabla_{E_{2}} E_{1}=0, & \nabla_{E_{3}} E_{1}=0 .
\end{array}
$$

The tangent vectors $X$ and $Y$ to $M$ are expressed as linear combinations of $E_{1}, E_{2}, E_{3}$; that is, $X=\sum_{i=1}^{3} a_{i} E_{i}$ and $Y=$ $\sum_{i=1}^{3} b_{i} E_{i}, a_{i}, b_{i}(i=1,2,3)$ are scalars. Clearly $M$ is a $\beta$ Kenmotsu manifold with $\beta=k$.

\section{Ricci Soliton in Trans-Sasakian Manifolds Satisfying $R(\xi, X) \cdot \widetilde{C}=0$}

The quasiconformal curvature tensor $\widetilde{C}$ is defined by

$\widetilde{C}(X, Y) Z=a R(X, Y) Z+b[S(Y, Z) X-S(X, Z) Y$

$$
\begin{array}{r}
+g(Y, Z) Q X-g(X, Z) Q Y] \\
-\frac{r}{n}\left(\frac{a}{n-1}+2 b\right)[g(Y, Z) X-g(X, Z) Y],
\end{array}
$$

where $a, b$ are constants. Taking $Z=\xi$ in (34) and using (10), (15), (16), and (17), we get

$$
\begin{aligned}
\widetilde{C}(X, Y) \xi= & {\left[a\left(\alpha^{2}-\beta^{2}\right)-b(2 \lambda+\beta)-\frac{r}{n}\left(\frac{a}{n-1}+2 b\right)\right] } \\
& \cdot[\eta(Y) X-\eta(X) Y]+2 \alpha \beta a \\
& \cdot[\eta(Y) \phi X-\eta(X) \phi Y] .
\end{aligned}
$$

Similarly using (12), (15), (16), and (17) in (34), we get

$$
\begin{aligned}
\eta(\widetilde{C}(X, Y) Z) \\
=\left[a\left(\alpha^{2}-\beta^{2}\right)-b(2 \lambda+\beta)-\frac{r}{n}\left(\frac{a}{n-1}+2 b\right)\right] \\
\cdot[g(Y, Z) \eta(X)-g(X, Z) \eta(Y)]-2 \alpha \beta a \\
\cdot[g(\phi X, Z) \eta(Y)-g(\phi Y, Z) \eta(X)] .
\end{aligned}
$$

We assume that the condition $R(\xi, X) \cdot \widetilde{C}=0$; then we have

$$
\begin{aligned}
& R(\xi, X) \widetilde{C}(Y, Z) W-\widetilde{C}(R(\xi, X) Y, Z) W \\
& -\widetilde{C}(Y, R(\xi, X) Z) W-\widetilde{C}(Y, Z) R(\xi, X) W=0,
\end{aligned}
$$


for all vector fields $X, Y, Z$, and $W$ on $M$. Using (10) in (37), we have

$$
\begin{aligned}
\left(\alpha^{2}-\beta^{2}\right)[g(\widetilde{C}(Y, Z) W, X) \xi-\eta(\widetilde{C}(Y, Z) W) X] \\
+2 \alpha \beta[g(\phi \widetilde{C}(Y, Z) W, X) \xi+\eta(\widetilde{C}(Y, Z) W) \phi X] \\
+(\operatorname{grad} \alpha) g(\phi \widetilde{C}(Y, Z) W, X)-(\operatorname{grad} \beta) \\
\cdot g(\phi \widetilde{C}(Y, Z) W, \phi X)-\left(\alpha^{2}-\beta^{2}\right) \\
\cdot[g(X, Y) \widetilde{C}(\xi, Z) W-\eta(Y) \widetilde{C}(X, Z) W]-2 \alpha \beta \\
\cdot[g(\phi Y, X) \widetilde{C}(\xi, Z) W+\eta(Y) \widetilde{C}(\phi X, Z) W] \\
-g(\phi Y, X) \widetilde{C}(\operatorname{grad} \alpha, Z) W+g(\phi X, \phi Y) \\
\cdot \widetilde{C}(\operatorname{grad} \beta, Z) W-\left(\alpha^{2}-\beta^{2}\right) \\
\cdot[g(X, Z) \widetilde{C}(Y, \xi) W-\eta(Z) \widetilde{C}(Y, X) W]-2 \alpha \beta \\
\cdot[g(\phi Z, X) \widetilde{C}(Y, \xi) W+\eta(Z) \widetilde{C}(Y, \phi X) W] \\
-g(\phi Z, X) \widetilde{C}(Y, \operatorname{grad} \alpha) W+g(\phi X, \phi Z) \\
. \widetilde{C}(Y, \operatorname{grad} \beta) W-\left(\alpha^{2}-\beta^{2}\right) \\
\cdot[g(X, W) \widetilde{C}(Y, Z) \xi-\eta(W) \widetilde{C}(Y, Z) X]-2 \alpha \beta \\
\cdot[g(\phi W, X) \widetilde{C}(Y, Z) \xi+\eta(W) \widetilde{C}(Y, Z) \phi X] \\
-g(\phi W, X) \widetilde{C}(Y, Z) \operatorname{grad} \alpha \\
+g(\phi X, \phi W) \widetilde{C}(Y, Z) \operatorname{grad} \beta=0 .
\end{aligned}
$$

By taking an inner product with $\xi$, we get

$$
\begin{gathered}
\left(\alpha^{2}-\beta^{2}\right)[g(\widetilde{C}(Y, Z) W, X)-\eta(\widetilde{C}(Y, Z) W) \eta(X)] \\
-\left(\alpha^{2}-\beta^{2}\right)[g(X, Y) \eta(\widetilde{C}(\xi, Z) W) \\
-\eta(Y) \eta(\widetilde{C}(X, Z) W)]-2 \alpha \beta \\
\cdot[g(\phi Y, X) \eta(\widetilde{C}(\xi, Z) W)+\eta(Y) \eta(\widetilde{C}(\phi X, Z) W)] \\
-g(\phi Y, X) \eta(\widetilde{C}(\operatorname{grad} \alpha, Z) W)+g(\phi X, \phi Y) \\
\cdot \eta(\widetilde{C}(\operatorname{grad} \beta, Z) W)-\left(\alpha^{2}-\beta^{2}\right) \\
\cdot[g(X, Z) \eta(\widetilde{C}(Y, \xi) W)-\eta(Z) \eta(\widetilde{C}(Y, X) W)]-2 \alpha \beta \\
\cdot[g(\phi Z, X) \eta(\widetilde{C}(Y, \xi) W)+\eta(Z) \eta(\widetilde{C}(Y, \phi X) W)] \\
-g(\phi Z, X) \eta(\widetilde{C}(Y, \operatorname{grad} \alpha) W)+g(\phi X, \phi Z) \\
\cdot \eta(\widetilde{C}(Y, \operatorname{grad} \beta) W)-\left(\alpha^{2}-\beta^{2}\right)
\end{gathered}
$$

$$
\begin{aligned}
& \cdot[g(X, W) \eta(\widetilde{C}(Y, Z) \xi)-\eta(W) \eta(\widetilde{C}(Y, Z) X)] \\
& -2 \alpha \beta[g(\phi W, X) \eta(\widetilde{C}(Y, Z) \xi)+\eta(W) \eta(\widetilde{C}(Y, Z) \phi X)] \\
& -g(\phi W, X) \eta(\widetilde{C}(Y, Z) \operatorname{grad} \alpha) \\
& +g(\phi X, \phi W) \eta(\widetilde{C}(Y, Z) \operatorname{grad} \beta)=0 .
\end{aligned}
$$

In view of (34), (35), and (36) and Remark 2 in (39), then we have

$$
\begin{gathered}
\left(\alpha^{2}-\beta^{2}\right)[g(R(Y, Z) W, X) \\
+b[S(Z, W) g(Y, X)-S(Y, W) g(Z, X) \\
+g(Z, W) S(Y, X)-g(Y, W) S(Z, X)] \\
-\frac{r}{n}\left(\frac{a}{n-1}+2 b\right) \\
\cdot[g(Z, W) g(Y, X)-g(Y, W) g(Z, X)]] \\
+\left(\alpha^{2}-\beta^{2}\right)\left[a\left(\alpha^{2}-\beta^{2}\right)-b(2 \lambda+\beta)-\frac{r}{n}\left(\frac{a}{n-1}+2 b\right)\right] \\
\cdot[g(X, Z) g(Y, W)-g(X, Y) g(Z, W)]+2 \alpha \beta a\left(\alpha^{2}-\beta^{2}\right) \\
\cdot[g(X, Z) g(\phi Y, W)-g(\phi Y, X) \eta(W) \eta(Z)+g(\phi Z, X) \\
\cdot \eta(Y) \eta(W)]+2 \alpha \beta \\
{\left[a\left(\alpha^{2}-\beta^{2}\right)-b(2 \lambda+\beta)-\frac{r}{n}\left(\frac{a}{n-1}+2 b\right)\right]} \\
\cdot[g(X, \phi Z) g(Y, W)-g(X, \phi Y) g(Z, W)]+4 \alpha^{2} \beta^{2} a \\
\cdot[g(\phi Z, X) g(\phi Y, W)+g(\phi Y, \phi X) \eta(W) \eta(Z) \\
-g(\phi Z, \phi X) \eta(Y) \eta(W)]=0 .
\end{gathered}
$$

Taking $X=Y=e_{i}$ in (40) and summing over $i=1,2, \ldots, n$, we get

$$
\begin{aligned}
& \left(\alpha^{2}-\beta^{2}\right)[a+b(n-2)] S(Z, W) \\
& +\left[b r\left(\alpha^{2}-\beta^{2}\right)-\frac{r\left(\alpha^{2}-\beta^{2}\right)(n-1)}{n}\left(\frac{a}{n-1}+2 b\right)\right] \\
& \cdot g(Z, W)-\left(\alpha^{2}-\beta^{2}\right)(n-1) \\
& \cdot\left[a\left(\alpha^{2}-\beta^{2}\right)-b(2 \lambda+\beta)-\frac{r}{n}\left(\frac{a}{n-1}+2 b\right)\right] \\
& \cdot g(Z, W)-2 \alpha \beta a\left(\alpha^{2}-\beta^{2}\right) g(\phi Z, W)+2 \alpha \beta \\
& \cdot\left[a\left(\alpha^{2}-\beta^{2}\right)-b(2 \lambda+\beta)-\frac{r}{n}\left(\frac{a}{n-1}+2 b\right)\right] \\
& \cdot g(\phi Z, W)+4 \alpha^{2} \beta^{2} a[-g(Z, W)+n \eta(Z) \eta(W)]=0 .
\end{aligned}
$$


Putting $Z=W=e_{i}$ in (41) and summing over $i=1,2, \ldots, n$, we get from (18)

$$
\begin{aligned}
\lambda[ & \left.-n(a+b(n-2))-n^{2} b+2 b n(n-1)\right] \\
& =(n-1) \beta(a+b(n-2))+a\left(\alpha^{2}-\beta^{2}\right) n(n-1) .
\end{aligned}
$$

Since by [12], a trans-Sasakian manifold of dimension $n \geq 5$ is either cosymplectic or $\alpha$-Sasakian or $\beta$-Kenmotsu manifold. So, based on this we state the following.

Theorem 3. A Ricci soliton $(g, \xi, \lambda)$ in a quasiconformally semisymmetric $\alpha$-Sasakian manifold is shrinking.

Proof. In (42) put $\beta=0$; then we have

$$
\begin{gathered}
\lambda\left[-n(a+b(n-2))-n^{2} b+2 b n(n-1)\right]=a \alpha^{2} n(n-1) \\
\Longrightarrow-a \lambda n=a \alpha^{2} n(n-1)
\end{gathered}
$$

On simplification we get $\lambda=-\alpha^{2}(n-1)$ and $\lambda<0$. Hence by Definition 1 Ricci soliton is shrinking.

Theorem 4. A Ricci soliton $(g, \xi, \lambda)$ in a quasiconformally semisymmetric $\beta$-Kenmotsu manifold is expanding.

Proof. In (42) put $\alpha=0$; then we have

$$
\begin{aligned}
-a \lambda n & =(n-1) \beta(a+b(n-2))-a \beta^{2} n(n-1) . \\
& \Longrightarrow-a \lambda n=-\beta(n-1)[-(a+b(n-2))+a \beta n] .
\end{aligned}
$$

If $a+b(n-2)=0$, then

$$
\lambda=\beta^{2}(n-1) .
$$

The above equation implies that $\lambda>0$. Hence Ricci soliton is expanding.

Theorem 5. A Ricci soliton $(g, \xi, \lambda)$ in a quasiconformally semisymmetric cosympletic manifold is steady.

Proof. In (42) put $\alpha=0$ and $\beta=0$; then we have

$$
-a \lambda n=0 .
$$

The above equation implies that $\lambda=0$. Hence Ricci soliton is steady.

\section{Ricci Soliton in Trans-Sasakian Manifolds Satisfying $P(\xi, X) \cdot \widetilde{C}=0$}

The projective curvature tensor is defined by

$$
P(X, Y) Z=R(X, Y) Z-\frac{1}{n-1}[S(Y, Z) X-S(X, Z) Y] .
$$

Putting $X=\xi$ in (47), we get

$$
P(\xi, Y) Z=R(X, Y) Z-\frac{1}{n-1}[S(Y, Z) X-S(X, Z) Y]
$$

We assume that $P(\xi, X) \cdot \widetilde{C}=0$; then we have

$$
\begin{aligned}
& P(\xi, X) \widetilde{C}(Y, Z) W-\widetilde{C}(P(\xi, X) Y, Z) W \\
& \quad-\widetilde{C}(Y, P(\xi, X) Z) W-\widetilde{C}(Y, Z) P(\xi, X) W=0 .
\end{aligned}
$$

Using (48) in (49), we obtain

$$
\begin{aligned}
\left(\alpha^{2}-\beta^{2}\right)[g(X, \widetilde{C}(Y, Z) W) \xi-\eta(\widetilde{C}(Y, Z) W) X]+2 \alpha \beta \\
\cdot[g(\phi \widetilde{C}(Y, Z) W, X) \xi+\eta(\widetilde{C}(Y, Z) W) \phi X]+(\operatorname{grad} \alpha) \\
\cdot g(\phi \widetilde{C}(Y, Z) W, X)-(\operatorname{grad} \beta) g(\phi \widetilde{C}(Y, Z) W, \phi X) \\
-\frac{1}{n-1}[-(\lambda+\beta) g(X, \widetilde{C}(Y, Z) W) \xi+\beta \eta(\widetilde{C}(Y, Z) W) \\
\cdot \eta(X) \xi+\lambda \eta(\widetilde{C}(Y, Z) W) X]-\left(\alpha^{2}-\beta^{2}\right) \\
\cdot[g(X, Y) \widetilde{C}(\xi, Z) W-\eta(Y) \widetilde{C}(X, Z) W]-2 \alpha \beta \\
\cdot[g(\phi Y, X) \widetilde{C}(\xi, Z) W+\eta(Y) \widetilde{C}(\phi X, Z) W]-g(\phi Y, X) \\
\cdot \widetilde{C}(\operatorname{grad} \alpha, Z) W+g(\phi X, \phi Y) \widetilde{C}(\operatorname{grad} \beta, Z) W+\frac{1}{n-1}
\end{aligned}
$$

$\cdot[-(\lambda+\beta) g(X, Y) \widetilde{C}(\xi, Z) W+\beta \eta(Y) \eta(X) \widetilde{C}(\xi, Z) W$

$$
\begin{aligned}
& +\lambda \eta(Y) \widetilde{C}(X, Z) W]-\left(\alpha^{2}-\beta^{2}\right) \\
\cdot & {[g(X, Z) \widetilde{C}(Y, \xi) W-\eta(Z) \widetilde{C}(Y, X) W]-2 \alpha \beta } \\
\cdot & {[g(\phi Z, X) \widetilde{C}(Y, \xi) W+\eta(Z) \widetilde{C}(Y, \phi X) W]-g(\phi Z, X) }
\end{aligned}
$$$$
\text { . } \widetilde{C}(Y, \operatorname{grad} \alpha) W+g(\phi X, \phi Z) \widetilde{C}(Y, \operatorname{grad} \beta) W+\frac{1}{n-1}
$$$$
\cdot[-(\lambda+\beta) g(X, Z) \widetilde{C}(Y, \xi) W+\beta \eta(Z) \eta(X) \widetilde{C}(Y, \xi) W
$$$$
+\lambda \eta(Z) \widetilde{C}(Y, X) W]-\left(\alpha^{2}-\beta^{2}\right)
$$

$\cdot[g(X, W) \widetilde{C}(Y, Z) \xi-\eta(W) \widetilde{C}(Y, Z) X]-2 \alpha \beta$

$\cdot[g(\phi W, X) \widetilde{C}(Y, Z) \xi+\eta(W) \widetilde{C}(Y, Z) \phi X]-g(\phi W, X)$

. $\widetilde{C}(Y, Z) \operatorname{grad} \alpha+g(\phi X, \phi W) \widetilde{C}(Y, Z) \operatorname{grad} \beta+\frac{1}{n-1}$

$\cdot[-(\lambda+\beta) g(X, W) \widetilde{C}(Y, Z) \xi+\beta \eta(W) \eta(X) \widetilde{C}(Y, Z) \xi$

$$
+\lambda \eta(W) \widetilde{C}(Y, Z) X]=0 .
$$


By taking an inner product with $\xi$ and by using Remark 2 in (50), then, we obtain

$$
\begin{aligned}
& {\left[\left(\alpha^{2}-\beta^{2}\right)+\frac{\lambda+\beta}{n-1}\right]} \\
& \cdot[g(X, \widetilde{C}(Y, Z) W)-\eta(\widetilde{C}(Y, Z) W) \eta(X)]-\left(\alpha^{2}-\beta^{2}\right) \\
& \cdot[g(X, Y) \eta(\widetilde{C}(\xi, Z) W)-\eta(Y) \eta(\widetilde{C}(X, Z) W)]-2 \alpha \beta \\
& \cdot[g(\phi Y, X) \eta(\widetilde{C}(\xi, Z) W)+\eta(Y) \eta(\widetilde{C}(\phi X, Z) W)] \\
& +\frac{1}{n-1}[-(\lambda+\beta) g(X, Y) \eta(\widetilde{C}(\xi, Z) W)+\beta \eta(Y) \\
& \quad \cdot \eta(X) \eta(\widetilde{C}(\xi, Z) W)+\lambda \eta(Y) \eta(\widetilde{C}(X, Z) W)] \\
& -\left(\alpha^{2}-\beta^{2}\right)[g(X, Z) \eta(\widetilde{C}(Y, \xi) W)-\eta(Z) \eta(\widetilde{C}(Y, X) W)] \\
& -2 \alpha \beta[g(\phi Z, X) \eta(\widetilde{C}(Y, \xi) W)+\eta(Z) \eta(\widetilde{C}(Y, \phi X) W)] \\
& +\frac{1}{n-1}[-(\lambda+\beta) g(X, Z) \eta(\widetilde{C}(Y, \xi) W)+\beta \eta(Z) \\
& \quad \eta \eta(X) \eta(\widetilde{C}(Y, \xi) W)+\lambda \eta(Z) \eta(\widetilde{C}(Y, X) W)] \\
& +\left(\alpha^{2}-\beta^{2}\right) \eta(W) \eta(\widetilde{C}(Y, Z) X)-2 \alpha \beta \eta(W) \\
& +\eta(\widetilde{C}(Y, Z) \phi X)+\frac{\lambda}{n-1} \eta(W) \eta(\widetilde{C}(Y, Z) X)=0 .
\end{aligned}
$$

By using (34), (35), and (36) in (51) and on simplification, we obtain

$$
\begin{aligned}
& {\left[\left(\alpha^{2}-\beta^{2}\right)+\frac{\lambda+\beta}{n-1}\right] \operatorname{ag}(X, R(Y, Z) W)+b} \\
& \cdot[S(Z, W) g(Y, X)-S(Y, W) g(Z, X) \\
& \quad+g(Z, W) S(Y, X)-g(Y, W) S(Z, X)] \\
& -\frac{r}{n}\left(\frac{a}{n-1}+2 b\right)[g(Z, W) g(Y, X)-g(Y, W) g(Z, X)] \\
& -\left[\left(\alpha^{2}-\beta^{2}\right)+\frac{\lambda+\beta}{n-1}\right] \\
& \cdot\left[a\left(\alpha^{2}-\beta^{2}\right) \eta(X)\{g(Z, W) \eta(Y)-g(Y, W) \eta(Z)\}\right. \\
& +2 \alpha \beta[g(\phi Z, W) \eta(Y)-g(\phi Y, W) \eta(Z)]+b \\
& \cdot[S(Z, W) \eta(Y) \eta(X)-S(Y, W) \eta(Z) \eta(X)+g(Z, W) \\
& \quad \cdot S(Y, \xi) \eta(X)-g(Y, W) S(Z, \xi) \eta(X)] \\
& \quad-\frac{r}{n}\left(\frac{a}{n-1}+2 b\right) \\
& \cdot[g(Z, W) \eta(Y) \eta(X)-g(Y, W) \eta(Z) \eta(X)]] \\
& +\left(\alpha^{2}-\beta^{2}\right) k
\end{aligned}
$$

$\cdot[g(Z, W) \eta(X) \eta(Y)-g(X, Y) g(Z, W)$

$$
+g(X, Z) g(Y, W)-g(Y, W) \eta(X) \eta(Z)]+2 \alpha \beta k
$$

$\cdot[g(\phi Z, X) g(Y, W)-g(\phi Y, X) g(Z, W)]+\frac{\lambda+\beta}{n-1} k$

$\cdot[g(X, Y) \eta(Z) \eta(W)-g(X, Y) g(Z, W)$

$+g(Z, W) \eta(X) \eta(Y)-g(X, Z) \eta(Y) \eta(W)$

$+g(X, Z) g(Y, W)-g(Y, W) \eta(X) \eta(Z)]+\frac{2 a \lambda \alpha \beta}{n-1}$

$\cdot[g(\phi Z, W) \eta(X) \eta(Y)+g(\phi Z, X) \eta(W) \eta(Y)$

$-g(\phi Y, X) \eta(W) \eta(Z)]+\frac{\lambda}{n-1} k$

$\cdot[g(X, Z) \eta(Y) \eta(W)-g(X, Y) \eta(Z) \eta(W)]+\frac{\lambda+\beta}{n-1}$

· $[2 a \alpha \beta\{g(X, Z) g(\phi Y, W)-g(\phi Y, W) \eta(X) \eta(Z)\}]$

$+2 a \lambda \alpha \beta\left(\alpha^{2}-\beta^{2}\right)$

$\cdot[g(X, Z) g(\phi Y, W)-g(\phi Y, W) \eta(X) \eta(Z)$

$+g(\phi Z, W) \eta(X) \eta(Y)-g(\phi Y, X) \eta(W) \eta(Z)$

$+g(\phi Z, X) \eta(Y) \eta(W)]+4 a \alpha^{2} \beta^{2}$

$\cdot[g(\phi Z, X) g(\phi Y, W)+g(\phi Y, \phi X) \eta(W) \eta(Z)$

$-g(\phi Z, \phi X) \eta(W) \eta(Y)]=0$,

where $k=\left[a\left(\alpha^{2}-\beta^{2}\right)-b(2 \lambda+\beta)-(r / n)(a /(n-1)+2 b)\right]$.

Taking $X=Y=e_{i}$ in (52) and summing over $i=$ $1,2, \ldots, n$, and on simplification, we obtain

$$
\begin{aligned}
& {\left[\left(\alpha^{2}-\beta^{2}\right)+\frac{\lambda+\beta}{n-1}\right](a+b(n-2)) S(Z, W)+\left(\alpha^{2}-\beta^{2}\right)} \\
& \cdot\left[b \lambda(n-2)-a\left(\alpha^{2}-\beta^{2}\right)(n-1)\right] g(Z, W)+\frac{\lambda+\beta}{n-1} \\
& \cdot\left[b \lambda(n-2)-a\left(\alpha^{2}-\beta^{2}\right)(n-1)\right] g(Z, W)+\frac{\beta}{n-1} \\
& \cdot\left[a\left(\alpha^{2}-\beta^{2}\right)-b(2 \lambda+\beta)-\frac{r}{n}\left(\frac{a}{n-1}+2 b\right)\right](n-1) \eta(Z) \\
& \cdot \eta(W)+2 \alpha \beta\left[a\left(\alpha^{2}-\beta^{2}\right)-b(2 \lambda+\beta)-\frac{r}{n}\left(\frac{a}{n-1}+2 b\right)\right] \\
& \cdot g(\phi Z, W)+\frac{2 a \alpha \beta \lambda}{n-1} g(\phi Z, W)+2 a \alpha \beta\left(\alpha^{2}-\beta^{2}\right) \\
& \cdot g(\phi Z, W)+4 a \alpha^{2} \beta^{2}[-g(Z, W)+n \eta(Z) \eta(W)]=0 .
\end{aligned}
$$


Taking $Z=W=e_{i}$ in (53) and summing over $i=1,2, \ldots, n$, and on simplification, we obtain

$$
\begin{aligned}
2 r(a+b(n-2)) & {\left[\left(\alpha^{2}-\beta^{2}\right)+\frac{\lambda+\beta}{n-1}\right]+2 n\left(\alpha^{2}-\beta^{2}\right) } \\
\cdot & {\left[b \lambda(n-2)-a\left(\alpha^{2}-\beta^{2}\right)(n-1)\right]+\frac{2 n(\lambda+\beta)}{n-1} } \\
\cdot & {\left[b \lambda(n-2)-a\left(\alpha^{2}-\beta^{2}\right)(n-1)\right]+\frac{2 \beta}{n-1} } \\
\cdot & {\left[a\left(\alpha^{2}-\beta^{2}\right)-b(2 \lambda+\beta)-\frac{r}{n}\left(\frac{a}{n-1}+2 b\right)\right] } \\
\cdot & (n-1)=0 .
\end{aligned}
$$

If $a+b(n-2)=0$ and on simplification, we get

$$
\begin{aligned}
n \lambda^{2} & +\lambda(n-1)\left[\beta+2 n\left(\alpha^{2}-\beta^{2}\right)\right] \\
& +(n-1)^{2}\left(\alpha^{2}-\beta^{2}\right)\left[n\left(\alpha^{2}-\beta^{2}\right)+\beta\right]=0 .
\end{aligned}
$$

Again by [12], a trans-Sasakian manifold of dimension $n \geq 5$ is either cosymplectic or $\alpha$-Sasakian or $\beta$-Kenmotsu manifold. So, based on this we state the following.

Theorem 6. A Ricci soliton in an $\alpha$-Sasakian manifold satisfying $P(\xi, X) \cdot \widetilde{C}=0$ is shrinking, provided $a+b(n-2)=0$.

Proof. In (55) put $\beta=0$; then we have

$$
\begin{gathered}
n \lambda^{2}+2 n \alpha^{2} \lambda(n-1)+n(n-1)^{2} \alpha^{4}=0 \\
\Longrightarrow\left[\lambda+(n-1) \alpha^{2}\right]^{2}=0,
\end{gathered}
$$

that is, $\lambda=-(n-1) \alpha^{2}$ and $\lambda<0$. This completes the proof of the theorem.

Theorem 7. A Ricci soliton in a $\beta$-Kenmotsu manifold satisfy$\operatorname{ing} P(\xi, X) \cdot \widetilde{C}=0$ is expanding, provided $a+b(n-2)=0$.

Proof. In (55) put $\alpha=0$; then we have

$$
n \lambda^{2}+\lambda(n-1)\left[\beta-2 n \beta^{2}\right]-(n-1)^{2} \beta^{2}\left[-n \beta^{2}+\beta\right]=0 .
$$

On simplifying the above quadratic equation then we get

$$
\lambda=\frac{-(n-1) \beta(1-2 n \beta) \pm(n-1) \beta}{2 n}
$$

that is either

$$
\begin{aligned}
& \lambda=\frac{-(n-1) \beta(1-2 n \beta)+(n-1) \beta}{2 n} \text { or } \\
& \lambda=\frac{-(n-1) \beta(1-2 n \beta)-(n-1) \beta}{2 n}
\end{aligned}
$$

on simplification we get

$$
\lambda=(n-1) \beta^{2} \quad \text { or } \quad \lambda=\frac{(n-1) \beta(n \beta-1)}{n} .
$$

In both cases $\lambda>0$. This completes the proof of the theorem.

Theorem 8. A Ricci soliton in a cosympletic manifolds satisfy$\operatorname{ing} P(\xi, X) \cdot \widetilde{C}=0$ is steady, provided $a+b(n-2)=0$.

Proof. In (55) put $\alpha=0$ and $\beta=0$; then we have

$$
n \lambda^{2}=0 \text {, }
$$

since $\lambda=0$. This completes the proof of the theorem.

\section{Ricci Soliton in Trans-Sasakian Manifolds Satisfying $H(\xi, X) \cdot S=0$}

Let $M$ be an $n$-dimensional trans-Sasakian manifolds admitting a Ricci soliton $(g, V, \lambda)$. The conharmonic curvature tensor on $M$ is given by

$$
\begin{aligned}
H(X, Y) Z \\
=R(X, Y) Z-\frac{1}{n-2} \\
\quad \cdot[S(Y, Z) X-S(X, Z) Y+g(Y, Z) Q X-g(X, Z) Q Y] .
\end{aligned}
$$

Putting $X=\xi$ in (62) and by using (10), (15), (16), and (17), we get

$$
\begin{aligned}
H(\xi, Y) Z= & \left(\alpha^{2}-\beta^{2}\right)[g(Y, Z) \xi-\eta(Z) Y] \\
& +2 \alpha \beta[g(\phi Y, Z) \xi+\eta(Z) \phi Y]+g(Y, \phi Z) \\
& \cdot(\operatorname{grad} \alpha)-g(\phi Y, \phi Z)(\operatorname{grad} \beta)-\frac{1}{n-2} \\
& \cdot[-(2 \lambda+\beta) g(Y, Z) \xi+(2 \lambda+\beta) \eta(Z) Y],
\end{aligned}
$$

$\eta(H(\xi, Y) Z)$

$$
\begin{aligned}
= & \left(\alpha^{2}-\beta^{2}\right)[g(Y, Z)-\eta(Z) \eta(Y)]-\frac{1}{n-2} \\
& \cdot[-(2 \lambda+\beta) g(Y, Z)+(2 \lambda+\beta) \eta(Z) \eta(Y)] .
\end{aligned}
$$

We assume that $H(\xi, X) \cdot S=0$ holds. Then we have

$$
S(H(\xi, X) Y, Z)+S(Y, H(\xi, X) Z)=0 .
$$

By using (15) in (64), then we have

$$
\begin{gathered}
\beta \eta(Z) \eta(H(\xi, X) Y)-(\lambda+\beta) g(H(\xi, X) Y, Z)-(\lambda+\beta) \\
\cdot g(Y, H(\xi, X) Z)+\beta \eta(Y) \eta(H(\xi, X) Z)=0,
\end{gathered}
$$

that is,

$$
\begin{aligned}
\beta & {[\eta(Z) \eta(H(\xi, X) Y)+\eta(Y) \eta(H(\xi, X) Z)] } \\
& =(\lambda+\beta)[g(H(\xi, X) Y, Z)+g(Y, H(\xi, X) Z)] .
\end{aligned}
$$


By using (63) in (66), we have

$$
\begin{aligned}
2 \eta(X) & \eta(Y) \eta(Z)\left[-\beta\left(\alpha^{2}-\beta^{2}\right)-\frac{\beta(2 \lambda+\beta)}{(n-2)}\right] \\
+ & {\left[\beta\left(\alpha^{2}-\beta^{2}\right)+\frac{\beta(2 \lambda+\beta)}{(n-2)}\right] } \\
\cdot & {[g(X, Z) \eta(Y)+g(X, Y) \eta(Z)]=0 . }
\end{aligned}
$$

Taking $X=Y=e_{i}$ and summing over $i=1,2, \ldots, n$, and by virtue of (18), we obtain

$$
\lambda=-\frac{\left(\alpha^{2}-\beta^{2}\right)(n-2)-\beta}{2}
$$

Again by [12], a trans-Sasakian manifold of dimension $n \geq 5$ is either cosymplectic or $\alpha$-Sasakian or $\beta$-Kenmotsu manifold. So, based on this we state the following.

Theorem 9. A Ricci soliton in an $\alpha$-Sasakian manifold satisfying $H(\xi, X) \cdot S=0$ is shrinking.

Proof. In (68) put $\beta=0$; then we have

$$
\lambda=-\frac{\alpha^{2}(n-2)}{2} .
$$

We get $\lambda=-\left(\alpha^{2}(n-2)\right) / 2<0$. This completes the proof of the theorem.

Theorem 10. A Ricci soliton in a $\beta$-Kenmotsu manifold satisfying $H(\xi, X) \cdot S=0$ is expanding if $\beta>0$ and shrinking if $\beta<0$.

Proof. In (68) put $\alpha=0$; then we have

$$
\lambda=\frac{\beta[\beta(n-2)+1]}{2} .
$$

If $\beta>0$, then $\lambda>0$ and Ricci soliton is expanding. If $\beta<0$, then $\lambda<0$ and Ricci soliton is shrinking. This completes the proof of the theorem.

Theorem 11. A Ricci soliton in a cosymplectic manifolds satisfying $H(\xi, X) \cdot S=0$ is steady.

Proof. In (68) put $\alpha=0$ and $\beta=0$; then we have

$$
\lambda=0 \text {. }
$$

This completes the proof of the theorem.

\section{Ricci Soliton in Trans-Sasakian Manifolds Satisfying $\widetilde{C}(\xi, X) \cdot S=0$}

We assume that $\widetilde{C}(\xi, X) \cdot S=0$ holds. Then we have

$$
S(\widetilde{C}(\xi, X) Y, Z)+S(Y, \widetilde{C}(\xi, X) Z)=0 .
$$

By using (15) in (72), then we have

$$
\begin{gathered}
\beta \eta(Z) \eta(\widetilde{C}(\xi, X) Y)-(\lambda+\beta) g(\widetilde{C}(\xi, X) Y, Z)-(\lambda+\beta) \\
\cdot g(Y, \widetilde{C}(\xi, X) Z)+\beta \eta(Y) \eta(\widetilde{C}(\xi, X) Z)=0,
\end{gathered}
$$

that is,

$$
\begin{aligned}
& \beta[\eta(Z) \eta(\widetilde{C}(\xi, X) Y)+\eta(Y) \eta(\widetilde{C}(\xi, X) Z)] \\
& =(\lambda+\beta)[g(\widetilde{C}(\xi, X) Y, Z)+g(Y, \widetilde{C}(\xi, X) Z)] .
\end{aligned}
$$

By using (63) in (74), we have

$$
\begin{aligned}
& {\left[a \beta\left(\alpha^{2}-\beta^{2}\right)-b \beta(2 \lambda+\beta)-\frac{r \beta}{n}\left[\frac{a}{(n-1)}+2 b\right]\right]} \\
& \quad \cdot[g(X, Z) \eta(Y)+g(X, Y) \eta(Z)-2 \eta(X) \eta(Y) \eta(Z)] \\
& =0 .
\end{aligned}
$$

Taking $X=Y=e_{i}$ and summing over $i=1,2, \ldots, n$, and if $a+b(n-2)=0$, and by virtue of (18), we obtain

$$
\lambda=-\left(\alpha^{2}-\beta^{2}\right)(n-1) .
$$

Again by [12], a trans-Sasakian manifold of dimension $n \geq 5$ is either cosymplectic or $\alpha$-Sasakian or $\beta$-Kenmotsu manifold. So, based on this we state the following.

Theorem 12. A Ricci soliton in an $\alpha$-Sasakian manifold satisfying $\widetilde{C}(\xi, X) \cdot S=0$ is shrinking, provided $a+b(n-2)=0$.

Proof. In (76) put $\beta=0$; then we have

$$
\lambda=-\alpha^{2}(n-1) .
$$

We get $\lambda=-\alpha^{2}(n-1)<0$. This completes the proof of the theorem.

Theorem 13. A Ricci soliton in a $\beta$-Kenmotsu manifold satisfying $\widetilde{C}(\xi, X) \cdot S=0$ is expanding, provided $a+b(n-2)=0$.

Proof. In (76) put $\alpha=0$; then we have

$$
\lambda=\beta^{2}(n-1)
$$

then the above equation implies that $\lambda>0$. This completes the proof of the theorem.

Theorem 14. A Ricci soliton in a cosymplectic manifolds satisfying $\widetilde{C}(\xi, X) \cdot S=0$ is steady, provided $a+b(n-2)=0$.

Proof. In (76) put $\alpha=0$ and $\beta=0$, then we have

$$
\lambda=0 \text {. }
$$

This completes the proof of the theorem. 


\section{Acknowledgment}

The authors are grateful to the referee for revising the paper.

\section{References}

[1] R. S. Hamilton, "Three-manifolds with positive Ricci curvature," Journal of Differential Geometry, vol. 17, no. 2, pp. 255-306, 1982.

[2] P. Topping, Lectures on the Ricci Flow, vol. 325 of London Mathematical Society Lecture Note Series, Cambridge University Press, Cambridge, UK, 2006.

[3] R. Sharma, "Certain results on $K$-contact and $(k, \mu)$-contact manifolds," Journal of Geometry, vol. 89, no. 1-2, pp. 138-147, 2008.

[4] B. Chow, P. Lu, and L. Ni, Hamilton's Ricci Flow, Graduate Studies in Mathematics, vol. 77, American Mathematical Society, Providence, RI, USA, 2006.

[5] C. Călin and M. Crasmareanu, "From the Eisenhart problem to Ricci solitons in $f$-Kenmotsu manifolds," Bulletin of the Malaysian Mathematical Sciences Society, vol. 33, no. 3, pp. 361$368,2010$.

[6] C. S. Bagewadi and G. Ingalahalli, "Ricci solitons in Lorentzian $\alpha$-Sasakian manifolds," Acta Mathematica, vol. 28, no. 1, pp. 5968, 2012.

[7] G. Ingalahalli and C. S. Bagewadi, "Ricci solitons in $\alpha$-Sasakain manifolds," ISRN Geometry, vol. 2012, Article ID 421384, 14 pages, 2012.

[8] M. M. Tripathi, "Ricci solitons in contact metric manifolds," http://arxiv.org/abs/0801.4222.

[9] H. G. Nagaraja and C. R. Premalatha, "Ricci solitons in Kenmotsu manifolds," Journal of Mathematical Analysis, vol. 3, no. 2, pp. 18-24, 2012.

[10] A. Gray and L. M. Hervella, "The sixteen classes of almost Hermitian manifolds and their linear invariants," Annali di Matematica Pura ed Applicata, vol. 123, pp. 35-58, 1980.

[11] J. A. Oubiña, "New classes of almost contact metric structures," Publicationes Mathematicae Debrecen, vol. 32, no. 3-4, pp. 187193, 1985.

[12] J. C. Marrero, "The local structure of trans-Sasakian manifolds," Annali di Matematica Pura ed Applicata, vol. 162, pp. 77-86, 1992.

[13] D. Janssens and L. Vanhecke, "Almost contact structures and curvature tensors," Kodai Mathematical Journal, vol. 4, no. 1, pp. $1-27,1981$.

[14] D. E. Blair and J. A. Oubiña, "Conformal and related changes of metric on the product of two almost contact metric manifolds," Publicacions Matemàtiques, vol. 34, no. 1, pp. 199-207, 1990.

[15] C. S. Bagewadi and Venkatesha, "Some curvature tensors on a trans-Sasakian manifold," Turkish Journal of Mathematics, vol. 31, no. 2, pp. 111-121, 2007.

[16] U. C. De and K. De, "On a class of three-dimensional TransSasakian manifolds," Communications of the Korean Mathematical Society, vol. 27, no. 4, pp. 795-808, 2012.

[17] U. C. De and M. M. Tripathi, "Ricci tensor in 3-dimensional trans-Sasakian manifolds," Kyungpook Mathematical Journal, vol. 43, no. 2, pp. 247-255, 2003.

[18] A. A. Shaikh, K. K. Baishya, and S. Eyasmin, "On D-homothetic deformation of trans-Sasakian structure," Demonstratio Mathematica, vol. 41, no. 1, pp. 171-188, 2008.
[19] H. G. Nagaraja, C. R. Premalatha, and G. Somashekhara, "On an $(\varepsilon, \delta)$-trans-Sasakian structure," Proceedings of the Estonian Academy of Sciences, vol. 61, no. 1, pp. 20-28, 2012.

[20] D. E. Blair, Contact Manifolds in Riemannian Geometry, vol. 509 of Lecture Notes in Mathematics, Springer, Berlin, Germany, 1976. 


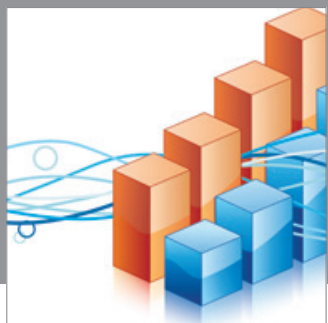

Advances in

Operations Research

mansans

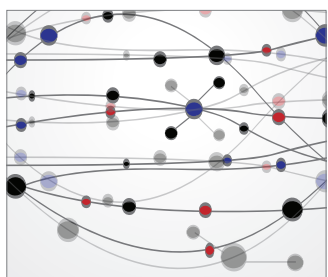

The Scientific World Journal
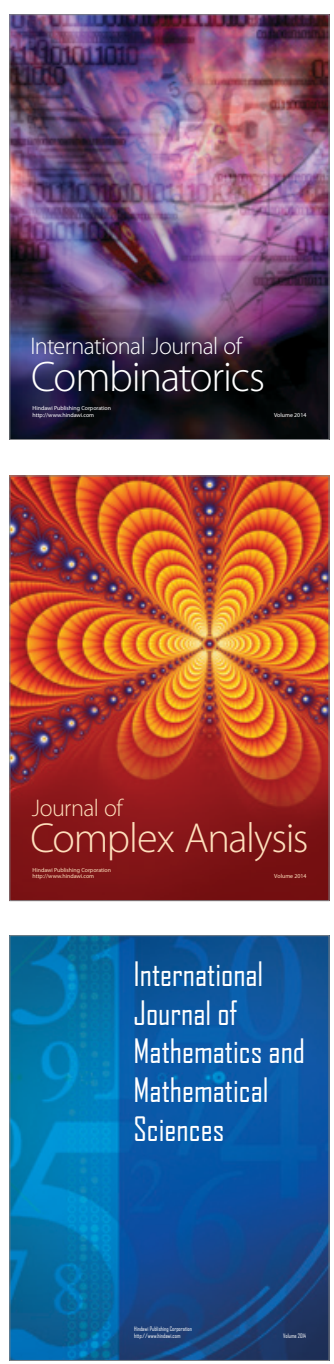
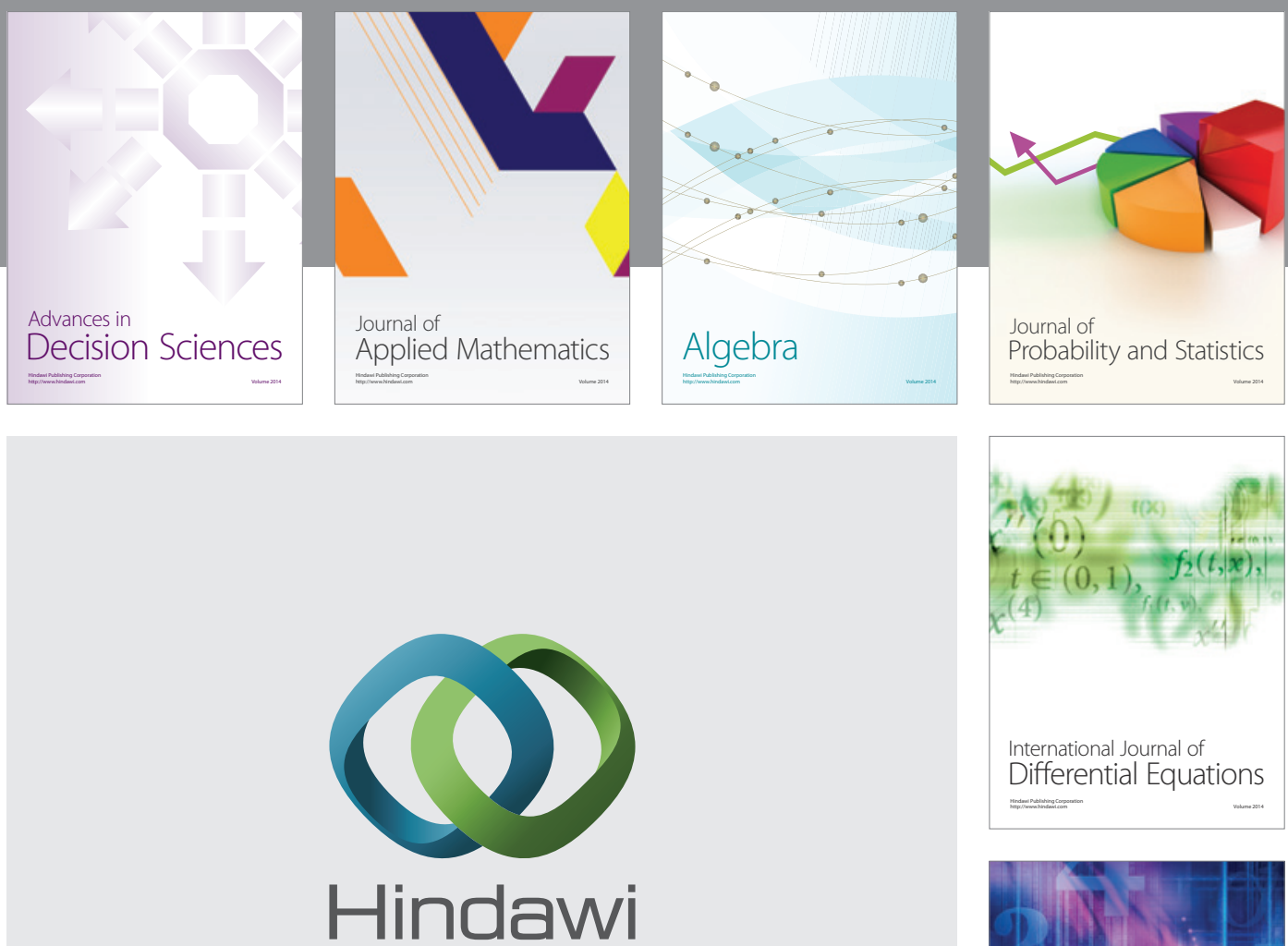

Submit your manuscripts at http://www.hindawi.com
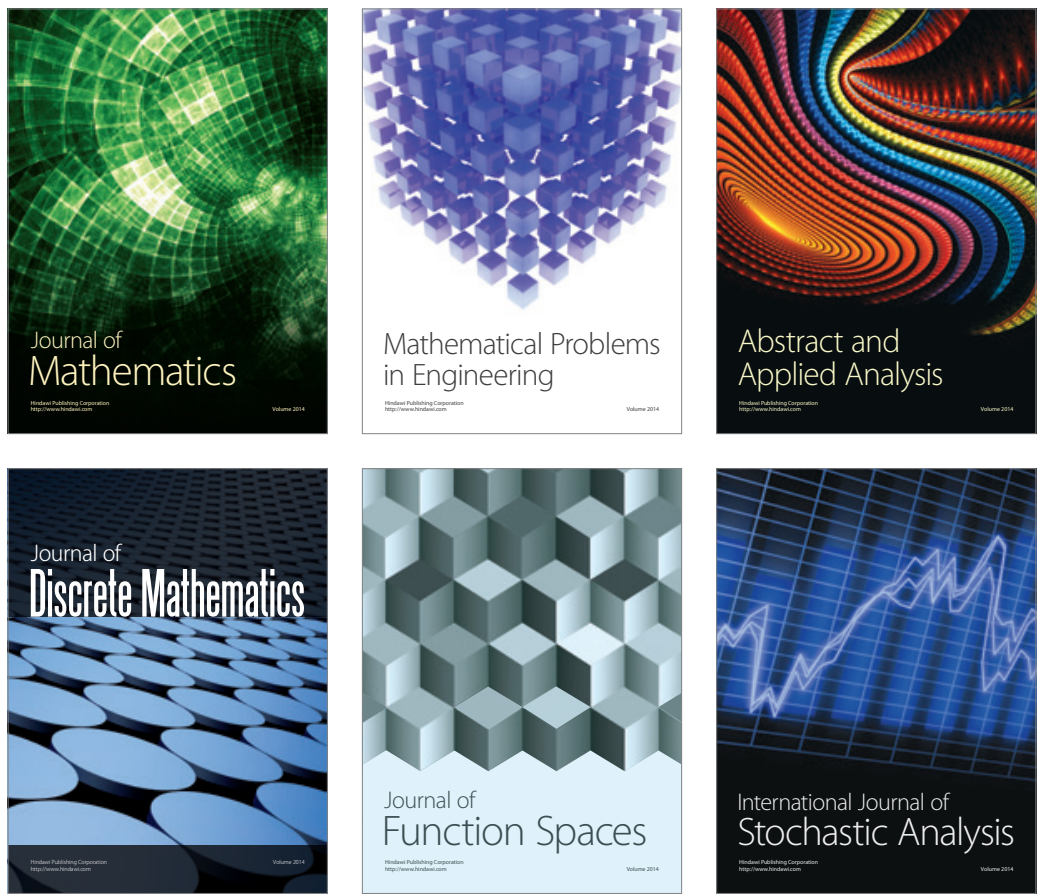

Journal of

Function Spaces

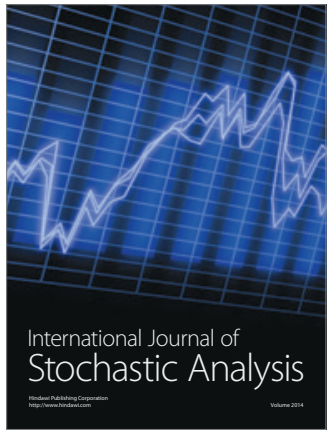

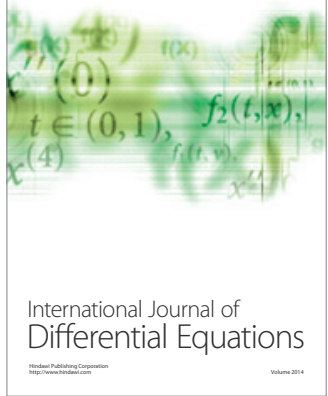
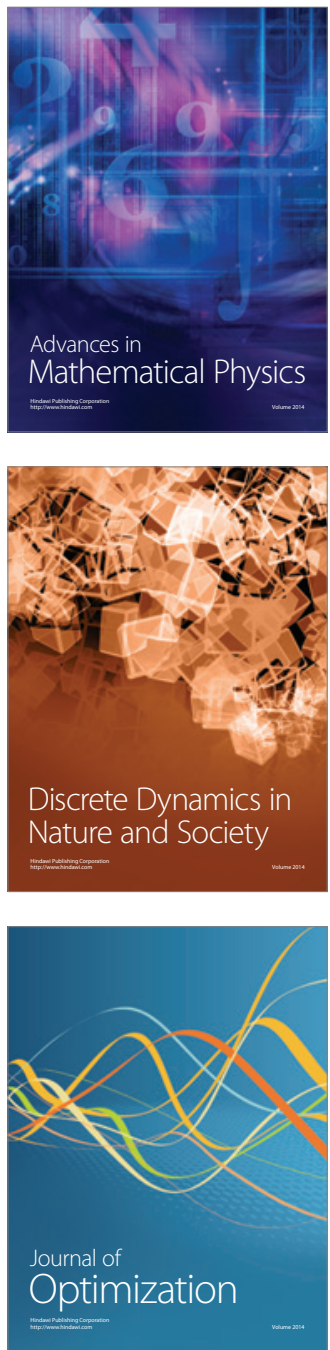